\title{
The Novel Uranyl Coordination Polymers Based on Quinoline-Containing Dicarboxylate by Altering Auxiliary Ligands: From 1D Chain to 3D Network
}

Kong-Qiu Hu †, Liu-Zheng Zhu †, , Cong-Zhi Wang, † Lei Mei, † YunHai Liu, $\neq$ Zeng-Qiang Gao †, Zhi-Fang Chai, \& and Wei-Qun Shi*,$\dagger$

\section{Supporting Information}

\author{
Table of contents
}

\section{S1. Scheme}

Scheme S1. The different bridging modes of bqde ${ }^{2-}$ ligand.

\section{S2. Figures}

Fig. S1. The EDS analyses of compound $\mathbf{3}$.

Fig. S2. The IR spectra of compounds $\mathbf{1}$ and $\mathbf{3}$.

Fig. S3. TGA of compounds $\mathbf{1}$ and $\mathbf{3}$ measured under air atmosphere with a heating rate of $5^{\circ} \mathrm{C} \cdot \mathrm{min}^{-1}$.

Fig. S4. Powder X-ray diffraction patterns of compound 1.

Fig. S5. Powder X-ray diffraction patterns of compound 3.

Fig. S6. Coordination geometries of hexacoordination uranyl complexes with different position atoms close to the equatorial plane : (a) 1,2 positions mode; (b) 1,4 positions mode; (c) 1,3 positions mode.

Fig. S7. (a) The 1D chain structure of compound 4. (b) The wave-shaped 2D structure of compound 4 formed by 1D chains via hydrogen bonds. (c) The hydrogen bonds between the 1D ligands. (d) The wave-shaped 2D layers associate with each other via $\pi \cdots \pi$ interactions to build the $3 \mathrm{D}$ stacking structure. 
Table S1. Selected Calculated and Experimental Bond Lengths $(\AA)$ of the U-O Bonds, Mayer Bond Order (MBO), and the Electron Density ( $\rho$, a.u.) at U-O and U-N Bond Critical Points (BCPs) for the Model Fragments of Compound $\mathbf{1}$.

Table S2. Selected Calculated and Experimental Bond Lengths $(\AA)$ of the U-O Bonds, MBO, and $\rho$ (a.u.) at U-O BCPs for the Model Fragments of Compound 2.

Table S3. Selected Calculated and Experimental Bond Lengths $(\AA)$ of the U-O Bonds, MBO, and $\rho$ (a.u.) at U-O BCPs for the Model Fragments of Compound 3.

Table S4. Percent Contribution of the U-O and U-N Bonding in Compounds 1, 2 and 3 .

S4. Structural description of compound 4 


\section{S1. Scheme}

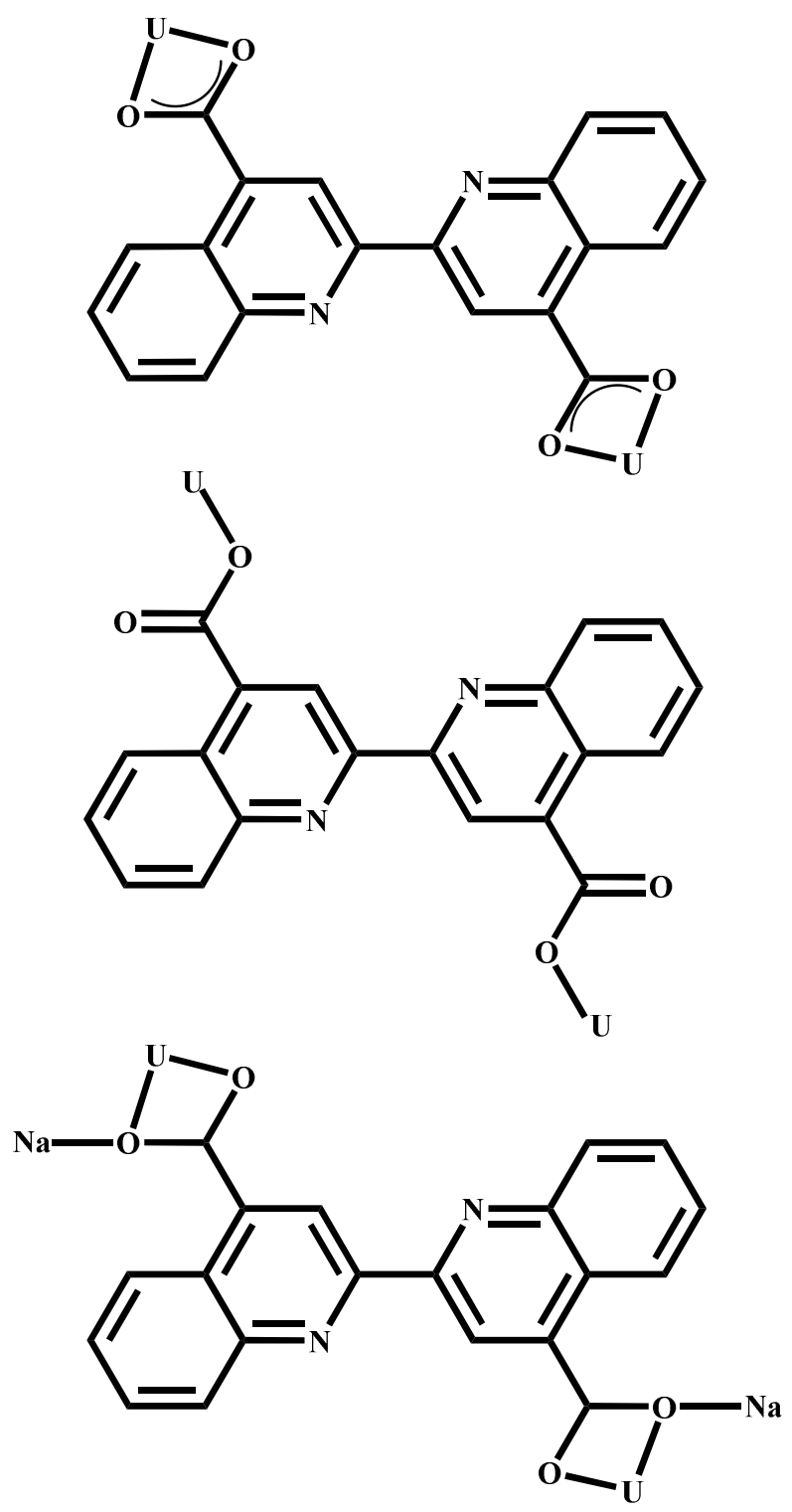

Scheme S1. The different bridging modes of bqde ${ }^{2-}$ ligand 


\section{S2. Figures}

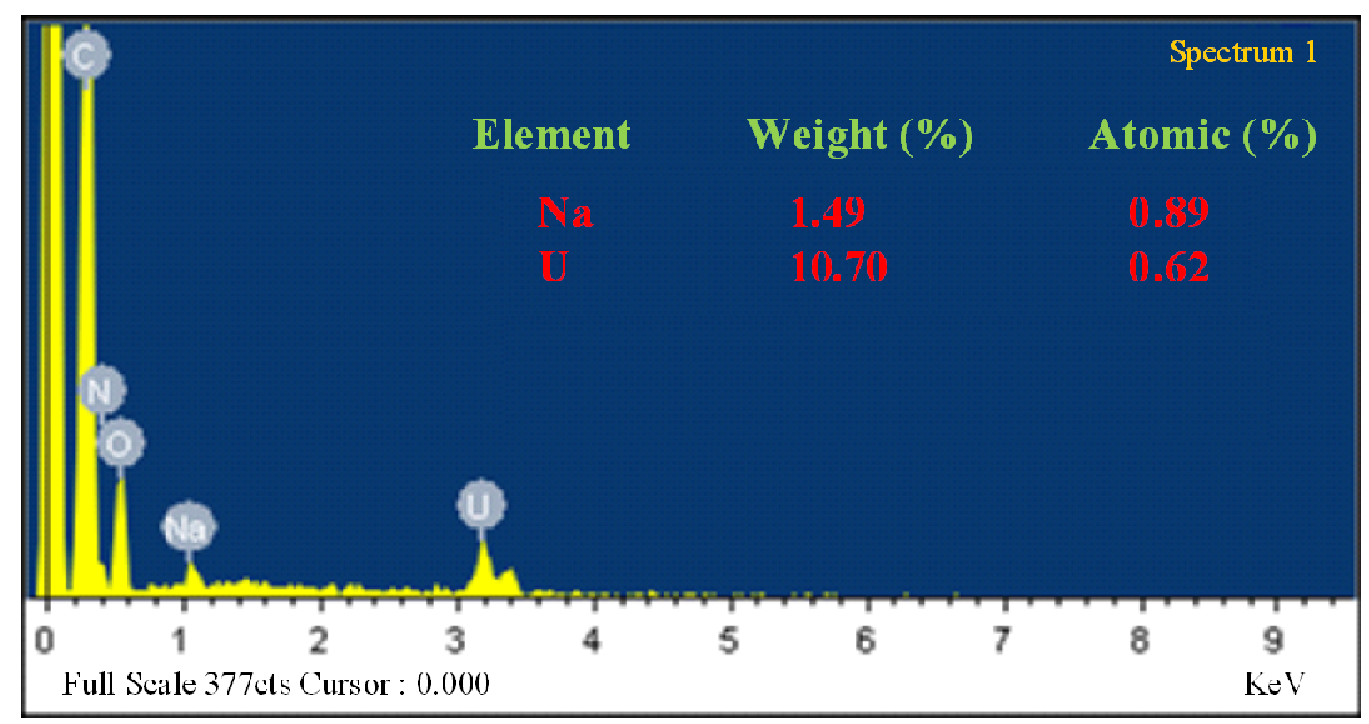

Fig. S1. The EDS analyses of compound 3

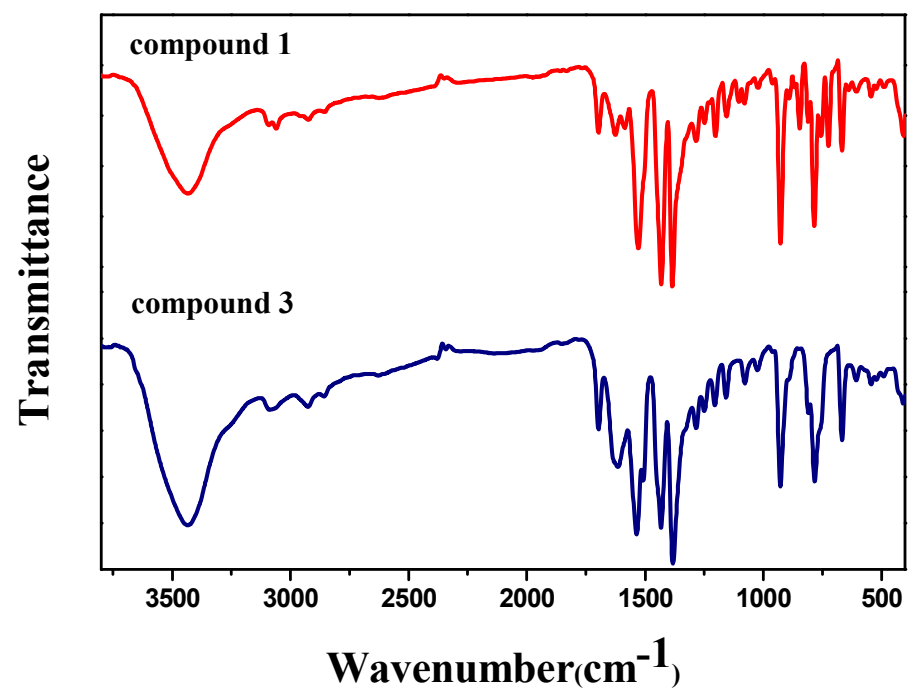

Fig. S2. The IR spectra of compounds 1 and 3 

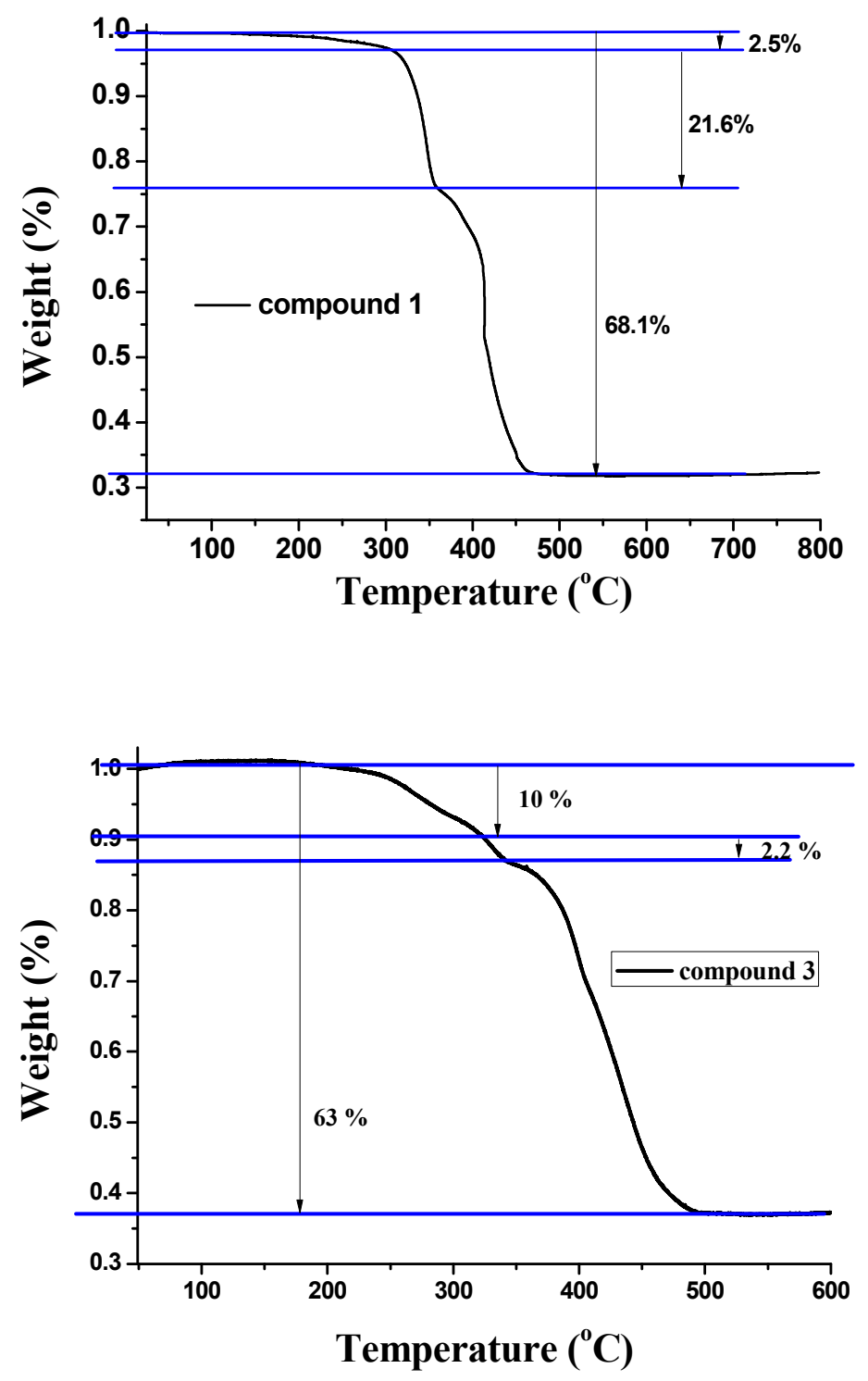

Fig. S3. TGA of compounds 1 and 3 measured under air atmosphere with a heating rate of $5^{\circ} \mathrm{C} \cdot \mathrm{min}^{-1}$ 


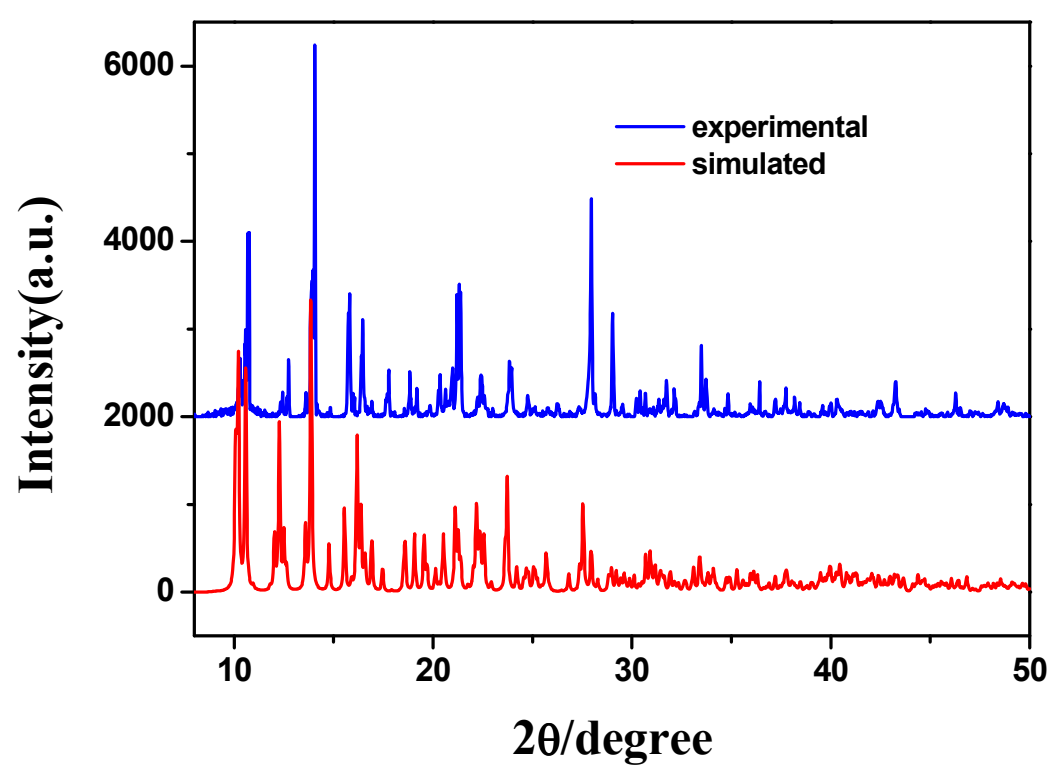

Fig. S4. Powder X-ray diffraction patterns of compound 1.

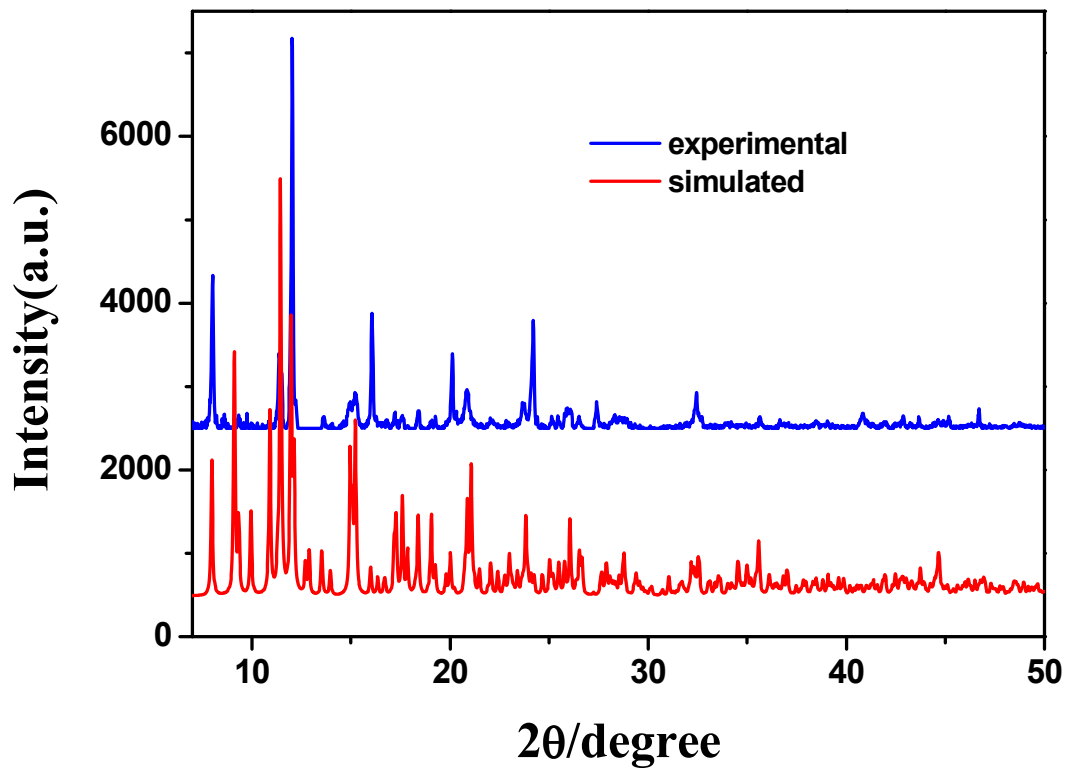

Fig. S5. Powder X-ray diffraction patterns of compound 3. 

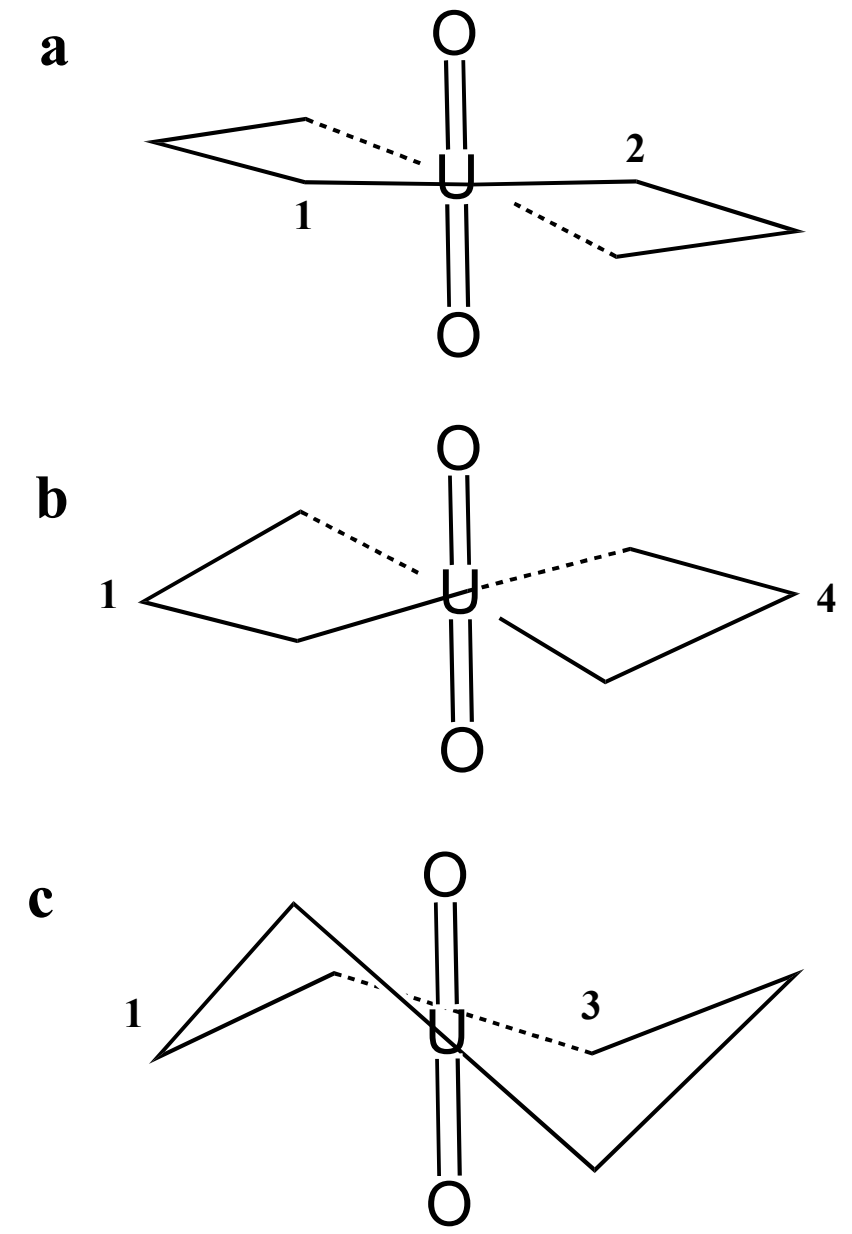

Fig. S6. Coordination geometries of hexacoordination uranyl compounds with different position atoms close to the equatorial plane: (a) 1,2 positions mode; (b) 1,4 positions mode; (c) 1,3 positions mode. 

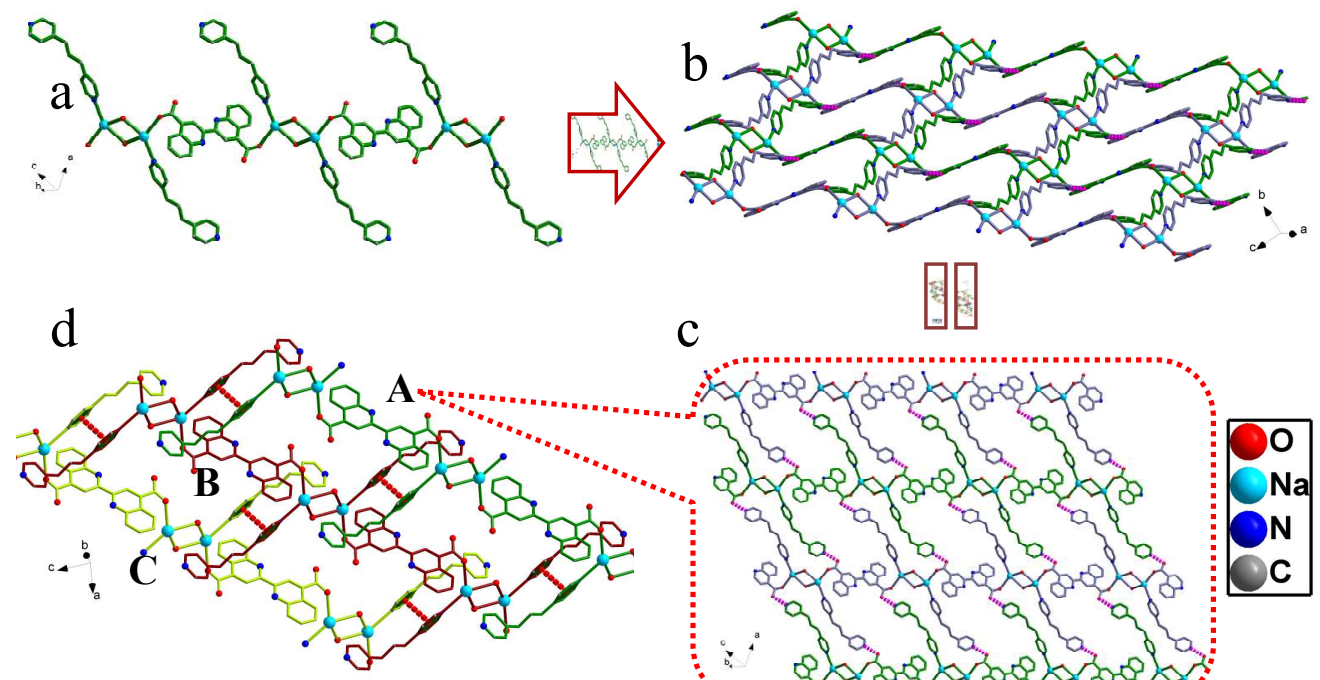

C
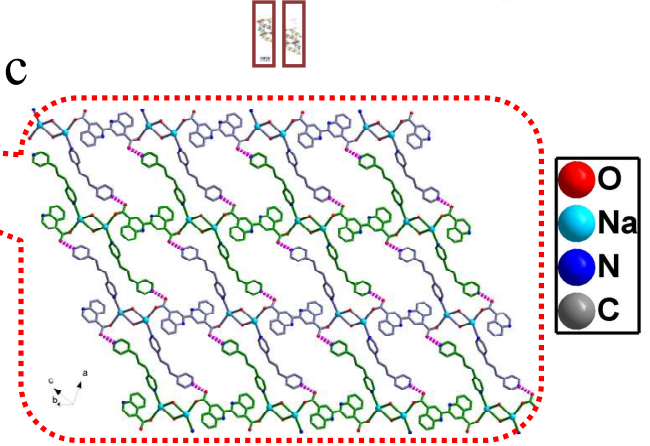

Fig. S7. (a) The 1D chain structure of compound 4. (b) The wave-shaped 2D structure of compound 4 formed by 1D chains via hydrogen bonds. (c) The hydrogen bonds between the 1D ligands. (d) The wave-shaped 2D layers associate with each other via $\pi \cdots \pi$ interactions to build the $3 \mathrm{D}$ stacking structure. 


\section{S3. Tables}

Table S1. Selected Calculated and Experimental Bond Lengths $(\AA)$ of the U-O Bonds, Mayer Bond Order (MBO), and the Electron Density ( $\rho$, a.u.) at U-O and U-N Bond Critical Points (BCPs) for the Model Fragments of Compound $\mathbf{1}$.

\begin{tabular}{|c|c|c|c|c|c|c|c|c|c|}
\hline Complex 1 & & $\mathrm{U} 1=\mathrm{O} 1$ & $\mathrm{U} 1=\mathrm{O} 2$ & U1-O3 & U1-O4 & U1-O5 & U1-O6 & U1-N1 & U1-N2 \\
\hline \multirow{2}{*}{$\begin{array}{l}\text { Bond } \\
\text { Lengths }\end{array}$} & Calc. & 1.785 & 1.785 & 2.509 & 2.451 & 2.483 & 2.484 & 2.669 & 2.663 \\
\hline & Exp. & $1.762(3)$ & $1.753(2)$ & $2.492(2)$ & $2.436(2)$ & $2.483(2)$ & $2.455(2)$ & $2.687(3)$ & $2.600(3)$ \\
\hline MBO & & 2.088 & 2.089 & 0.411 & 0.448 & 0.426 & 0.433 & 0.306 & 0.307 \\
\hline$\rho$ & & 0.2921 & 0.2919 & 0.0531 & 0.0604 & 0.0563 & 0.0563 & 0.0428 & 0.0434 \\
\hline
\end{tabular}

Table S2. Selected Calculated and Experimental Bond Lengths $(\AA)$ of the U-O Bonds, MBO, and $\rho$ (a.u.) at U-O BCPs for the Model Fragments of Compound 2.

\begin{tabular}{|c|c|c|c|c|c|c|c|c|}
\hline \multicolumn{2}{|c|}{ Complex 2} & $\mathrm{U} 1=\mathrm{O} 7$ & $\mathrm{U} 1=\mathrm{O} 8$ & U1-O4 & U1-O5w & U1-O6 & U1-O6' & U1-O9 \\
\hline \multirow{2}{*}{$\begin{array}{l}\text { Bond } \\
\text { Lengths }\end{array}$} & Calc & 1.798 & 1.797 & 2.407 & 2.555 & 2.371 & 2.369 & 2.356 \\
\hline & Exp. & $1.781(8)$ & $1.785(9)$ & $2.403(8)$ & $2.479(9)$ & $2.355(6)$ & $2.355(6)$ & $2.460(9)$ \\
\hline \multicolumn{2}{|l|}{ MBO } & 2.085 & 2.056 & 0.462 & 0.382 & 0.498 & 0.499 & 0.500 \\
\hline \multirow[t]{2}{*}{$\rho$} & & 0.2823 & 0.2833 & 0.0609 & 0.0466 & 0.0706 & 0.0714 & 0.0693 \\
\hline & & $\mathrm{U} 1^{\prime}=\mathrm{O} 7^{\prime}$ & $\mathrm{U} 1^{\prime}=\mathrm{O} 8^{\prime}$ & U1'-O4' & U1'-O5w' & U1'-O6 & U1'-O6' & U1'-O9' \\
\hline \multirow{2}{*}{$\begin{array}{l}\text { Bond } \\
\text { Lengths }\end{array}$} & Calc & 1.798 & 1.796 & 2.409 & 2.554 & 2.368 & 2.369 & 2.353 \\
\hline & Exp. & $1.781(8)$ & $1.785(9)$ & $2.403(8)$ & $2.479(9)$ & $2.355(6)$ & $2.355(6)$ & $2.460(9)$ \\
\hline \multicolumn{2}{|l|}{ MBO } & 2.066 & 2.072 & 0.461 & 0.383 & 0.498 & 0.496 & 0.502 \\
\hline \multicolumn{2}{|l|}{$\rho$} & 0.2823 & 0.2835 & 0.0607 & 0.0467 & 0.0712 & 0.0709 & 0.0697 \\
\hline
\end{tabular}


Table S3. Selected Calculated and Experimental Bond Lengths $(\AA)$ of the U-O Bonds, MBO, and $\rho$ (a.u.) at U-O BCPs for the Model Fragments of Compound 3.

\begin{tabular}{|c|c|c|c|c|c|c|c|c|c|}
\hline Complex $\mathbf{3}$ & & $\mathrm{U} 1=\mathrm{O} 7$ & $\mathrm{U} 1=\mathrm{O} 8$ & U1-O1 & U1-O2 & U1-O3 & U1-O4 & U1-O9 & U1-O10 \\
\hline \multirow{2}{*}{$\begin{array}{l}\text { Bond } \\
\text { Lengths }\end{array}$} & Calc. & 1.790 & 1.790 & 2.484 & 2.488 & 2.477 & 2.493 & 2.483 & 2.506 \\
\hline & Exp. & $1.765(10)$ & $1.754(9)$ & $2.503(8)$ & $2.472(7)$ & $2.371(10)$ & $2.480(8)$ & $2.483(10)$ & $2.484(9)$ \\
\hline MBO & & 2.078 & 2.082 & 0.435 & 0.423 & 0.426 & 0.434 & 0.426 & 0.433 \\
\hline$\rho$ & & 0.2881 & 0.2881 & 0.0562 & 0.0556 & 0.0570 & 0.0550 & 0.0563 & 0.0535 \\
\hline
\end{tabular}

Table S4. Percent Contribution of the U-O and U-N Bonding in Compounds 1, 2 and 3.

\begin{tabular}{|c|c|c|c|c|c|c|c|}
\hline \multirow{2}{*}{ Species } & \multirow{2}{*}{ Orbitals } & \multirow{2}{*}{ Element } & \multicolumn{5}{|c|}{ Contributions of Each Atomic Orbital (\%) } \\
\hline & & & $2 p$ & $5 f$ & $6 \mathrm{~d}$ & $7 \mathrm{~s}$ & $7 \mathrm{p}$ \\
\hline \multirow{17}{*}{ Complex 1} & \multirow{9}{*}{$\mathrm{c}$} & $\mathrm{U} 1$ & & 10 & & & 1 \\
\hline & & O5 & 14 & & & & \\
\hline & & O6 & 13 & & & & \\
\hline & & $\mathrm{O} 4$ & 11 & & & & \\
\hline & & N1 & 9 & & & & \\
\hline & & $\mathrm{O} 3$ & 8 & & & & \\
\hline & & $\mathrm{N} 2$ & 7 & & & & \\
\hline & & $\mathrm{O} 1$ & 2 & & & & \\
\hline & & $\mathrm{O} 2$ & 2 & & & & \\
\hline & \multirow{8}{*}{$\mathrm{d}$} & $\mathrm{U} 1$ & & 10 & 1 & 3 & 2 \\
\hline & & $\mathrm{O} 1$ & 15 & & & & \\
\hline & & O6 & 10 & & & & \\
\hline & & $\mathrm{O} 4$ & 6 & & & & \\
\hline & & $\mathrm{N} 2$ & 6 & & & & \\
\hline & & $\mathrm{O} 3$ & 4 & & & & \\
\hline & & O5 & 4 & & & & \\
\hline & & N1 & 2 & & & & \\
\hline \multirow{10}{*}{ Complex 2} & \multirow{4}{*}{ c } & U1' & & 2 & & & 2 \\
\hline & & O4' & 28 & & & & \\
\hline & & O9' & 7 & & & & \\
\hline & & O8' & 2 & & & & \\
\hline & \multirow{6}{*}{$\mathrm{d}$} & U1 & & 4 & & & \\
\hline & & U1' & & 3 & & & \\
\hline & & $\mathrm{O} 4$ & 18 & & & & \\
\hline & & O6 & 17 & & & & \\
\hline & & O9 & 7 & & & & \\
\hline & & O6' & 4 & & & & \\
\hline
\end{tabular}




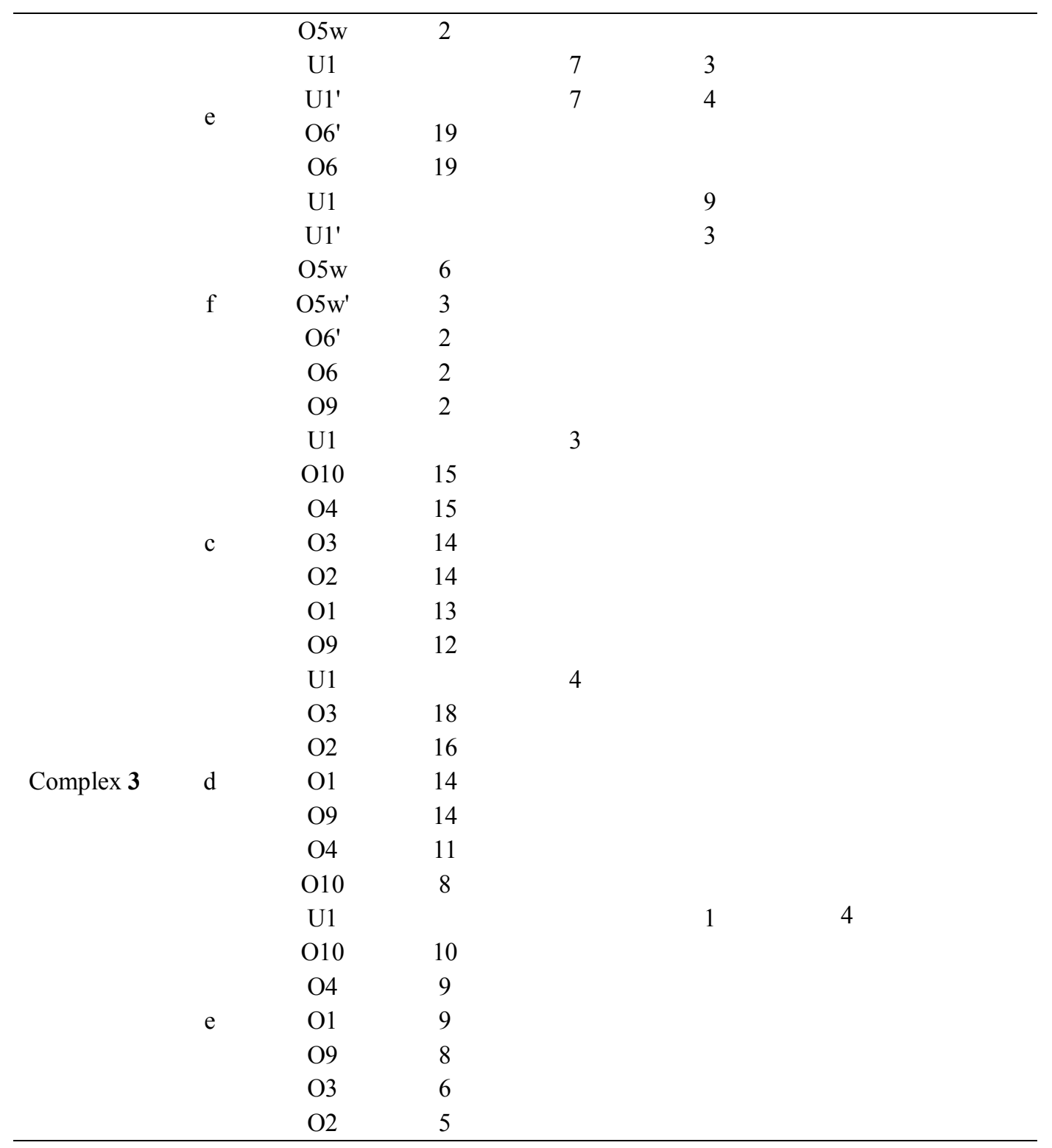

\section{S4. Structural description of compound 4}

Compound 4 crystallizes in a monoclinic space group $P \overline{1}$. The asymmetric unit of 4 consists of one sodium atom, half of a bqdc ${ }^{2-}$ ligand, one $\mathrm{N}$-containing auxiliary ligand (bpp), and one coordinated water molecule. As shown in Figure 1d, the sodium is coordinated with one $\mathrm{O}$ atom from the $\mathrm{bqdc}^{2-}$ ligand [the $\mathrm{Na}-\mathrm{O}$ bond length is 2.74(4) $\AA$ ], two $\mathrm{O}$ atoms from $\mathrm{H}_{2} \mathrm{O}$ ligands (the average bond length is $2.67 \AA$ ), and 
one $\mathrm{N}$ atom of bpp ligand. In compound 4 , the two identical sodium atoms are bridged by two $\mathrm{H}_{2} \mathrm{O}$ molecules to form a dinuclear $\mathrm{Na}_{2}$ cluster with a non-bonding $\mathrm{Na} \cdots \mathrm{Na}$ distance of $4.39(5) \AA$. The $\mathrm{Na}_{2}$ cluster is linked to each other by the bqde ${ }^{2-}$ ligand to generate a 1D chain structure (Fig. S7a). Meanwhile, the neighboring chains interconnect via hydrogen bonds (Fig. S7b, S7c) and face to face $\pi \cdots \pi$ interactions $[\mathrm{Cg} \cdots \mathrm{Cg}=3.61(1) \AA]$ between the pyridine rings of bpp ligands (Fig. S7d) to form 3D structure.

Further analysis of the stacking mode exposes the function of the intermolecular weak interaction during the process from 1D chain to 3D structure. As shown in Fig. S7c, a 1D chain (green) connects with two neighboring chains (gray) through the hydrogen bonds (pink dash line) to extend the wave-shaped 2D layer (Fig. S7b), which stack together to build the $3 \mathrm{D}$ structure, stabilized by the interfacial $\pi \cdots \pi$ interactions between layers (Fig. S7d). Having a close-up view of the packing mode in Fig. S7d, the brown 2D layer denoted by B, associate with two adjacent layers (A and C) via $\pi \cdots \pi$ interactions between the pyridine rings of bpp ligands. As described above, the phen ligand plays critical role in the formation of the complex 4 because of not only the role of complexant, but also, the vital function for the formation of the hydrogen bonds and $\pi \cdots \pi$ interactions. On the other hand, the intermolecular weak 
interaction facilitates stabilization of the flexible bpp ligand and avoids the disorder caused by atomic thermal vibration in the crystal lattice. 